\title{
Resistencia característica a compresión de una fábrica de ladrillo en función de la resistencia de sus componentes. Comprobación experimental de expresiones analíticas de la normativa europea
}

\section{Characteristic compression strength of a brickwork masonry starting from the strength of its components. Experimental verification of analitycal equations of european codes}

\begin{abstract}
Recepción/Received: 23-IX-05 Aceptación/Accepted: 13-II-06

\section{RESUMEN}

En este artículo se analiza la resistencia a compresión de una fábrica de ladrillo cerámico, asentado con mortero de cemento.

El objetivo es obtener la resistencia característica a compresión de la fábrica sin armar.

La investigación comprueba la fiabilidad de las expresiones analíticas existentes en la normativa europea, comparando la resistencia obtenida experimentalmente con la obtenida analíticamente, a partir de la resistencia de sus componentes (ladrillo cerámico y mortero de cemento).
\end{abstract}

A. Rolando $(*)$
Palabras clave: propiedades mecánicas, resistencia a compresión, ladrillo, cerámica, mortero.

\section{SUMMARY}

In this paper the compression strenght of a clay brickwork masonry binded with cement mortar is analyzed.

The target is to obtain the characteristic compression strength of unreinforced brickwork masonry.

This research try to test the validity of the analitycal equations in European codes, comparing the experimental strength with the analytically obtained from the strength of its components (clay brick and cement mortar).

Keywords: mechanical properties, compression strenght, brick, clay, cement mortar.

\section{INTRODUCCIÓN}

Diferentes normativas, tales como la española NBE FL-90 (5) que recoge experiencias previas (1-4), la británica BS 5628: Parte 1 (15), o la europea UNE-ENV-1996-1-1, indican que la resistencia característica de la fábrica de ladrillo a compresión puede ser medida a través de ensayos o calculada utilizando expresiones analíticas en forma de tablas o gráficos, que la determinan en función de las resistencias a compresión del ladrillo y del mortero, así como de la disposición o diseño constructivo de la fábrica.

En el libro La fábrica de ladrillo armada: una nueva tecnología aplicada a un material tradicional (6) realicé unos gráficos ajustando los valores que aparecen en la

(*) Dpto. de Construcción y Tecnología Arquitectónicas, E.T.S. de Arquitectura, Universidad Politécnica de Madrid (España). 
norma NBE FL-90 y en la norma UNE-ENV-1996-1-1. En estos gráficos se puede obtener directamente la resistencia característica de la fábrica de ladrillo a compresión en función de las resistencias de sus componentes y de las características constructivas. En esta publicación se propone, igualmente, una expresión que ajusta los coeficientes de las expresiones analíticas dadas por la UNE-ENV-1996-1-1 a las experiencias españolas recogidas en la NBE FL-90, presentando únicamente la dificultad de definir en términos europeos los conceptos dados para el mortero de: magro, sograso y graso.

La obtención de expresiones analíticas fiables y ajustadas a los materiales y forma de construir de cada país (7) es fundamental para el cálculo, por otra parte, cada vez más informatizado. Estas iniciativas equiparan el sistema de cálculo de las fábricas al de otros materiales.

El objeto de esta investigación es comprobar, de forma experimental para el entorno de la construcción de fábrica de ladrillo en España, la fiabilidad de las expresiones analíticas dadas por la norma UNE-ENV-1996-1-1: "Eurocódigo 6. Proyecto de estructuras de fábrica. Parte 1-1: reglas generales para edificios. Reglas para fábrica y fábrica armada" (8).

\section{PARTE EXPERIMENTAL}

Se utiliza ladrillo cerámico perforado cara vista de Hermanos Díaz Redondo, S.A. Cobeja (Toledo), de dimensiones: 240x114x48 mm. Las dimensiones de las probetas de ladrillo refrentado son: $240 \times 114 \times 68 \mathrm{~mm}$.

La máquina de ensayo cumple con lo especificado en la norma UNE-EN-772-1. Métodos de ensayo para piezas de fábrica (10).

\section{Ladrillo}

Los ensayos se realizan de acuerdo con las normas UNEEN 772-1. Métodos de ensayo para piezas de fábrica. Parte 1: Determinación de la resistencia a compresión y UNE-EN 771-1 especificación para las piezas de fábrica. Parte 1: Piezas cerámicas (10) (9).

Para el refrentado de los ladrillos se utiliza un mortero de dosificación 1:1 (Cemento:Arena), utilizando cemento CEM-II/A-S 32,5 y una relación agua/cemento de 0,35, de tal forma que garantice una resistencia a compresión mínima, determinada en el momento de ensayar los ladrillos que sea, al menos, la resistencia esperada del ladrillo, en nuestro caso de 15 a $20 \mathrm{~N} / \mathrm{mm}^{2}$.

Las probetas se dejan endurecer durante 28 días en una cámara acondicionada al $90 \%$ de humedad relativa.

La Tabla 1 recoge los resultados de los ensayos de resistencia a compresión de las probetas de ladrillo. La resistencia normalizada a compresión es de $16 \mathrm{~N} / \mathrm{mm}^{2}$. En la ficha de características técnicas del ladrillo, el fabricante garantiza una resistencia a compresión de $15 \mathrm{~N} / \mathrm{mm}^{2}$, frente a los $10 \mathrm{~N} / \mathrm{mm}^{2}$ exigidos. Los ensayos demuestran que cumple perfectamente.

\section{Mortero}

Del mismo mortero empleado para la fabricación de la probeta de fábrica en muretes, se extraen moldes con tres probetas prismáticas por amasada/día de dimensiones: $40 \times 40 \times 40 \mathrm{~mm}$.

Los aparatos, la preparación de la muestra y la conservación de las probetas de ensayo, se realizaron de acuerdo con las normas UNE-EN 998-2. Especificaciones de los morteros para albañilería. Parte 2: Morteros para albañilería; UNE-EN 1015-11. Métodos de ensayo de los morteros

Tabla 1

Resultado de los ensayos a compresión, según la norma UNE-EN 772-1 de las probetas de ladrillo

\begin{tabular}{|c|c|c|}
\hline Probeta & Carga (kg) & Tensión $\left(\mathbf{N} / \mathbf{m m}^{\mathbf{2}}\right)$ \\
\hline 1 & 69.500 & 25,92 \\
\hline 2 & 55.000 & 20,51 \\
\hline 3 & 61.500 & 22,93 \\
\hline 4 & 49.500 & 18,46 \\
\hline 5 & 57.500 & 21,44 \\
\hline 6 & 75.000 & 27,97 \\
\hline 7 & 59.500 & 22,19 \\
\hline V. Medio & & 22,77 \\
\hline Factor de forma $\delta$ & $\mathbf{0 , 7}$ & $\mathbf{1 6}$ \\
\hline Resistencia normalizada $(\delta *$ V. Medio $)$ & & \\
\hline
\end{tabular}

$1 \delta$ - Factor de forma que permite tener en cuenta las dimensiones de las probetas sometidas a ensayos después de la preparación de su superficie. Aparece en la Norma UNE-EN 772-1, Tabla A.1 para una probeta de ladrillo refrenando de altura $68 \mathrm{~mm}$ y anchura $240 \mathrm{~mm}$. 
para albañilería. Parte 11: Determinación de la resistencia a flexión y a compresión del mortero endurecido; UNE-EN 1015-2. Métodos de ensayo de los morteros para albañilería. Parte 2: Toma de muestras total de morteros y preparación de los morteros para ensayo (11-13).

Los ensayos se realizaron de acuerdo a la norma UNE-EN 1015-11. Métodos de ensayo de los morteros para albañilería. Parte 11: Determinación de la resistencia a flexión y a compresión del mortero endurecido.

Para el mortero de juntas se utilizó cemento CEM-II/A-S 32,5 , arena de río de $\phi=5$, de acuerdo a las condiciones de granulometría de una arena que se establecen en la Tabla 2, Figura 1 del apartado 3.1.3. Arenas para los morteros de las fábricas según la norma NBE FL-90. Muros resistentes de fábrica de ladrillo, dosificación 1:4 de cemento:arena, relación agua/cemento de 0,5 y curado en la misma nave de ensayos, cubiertas con plástico a 28 días. La Figura 1 muestra el análisis granulométrico de la arena empleada en la fabricación de los morteros. Se comprueba que su granulometría está dentro de los valores especificados en la norma NBE FL-90.

La Tabla 2 recoge los resultados de los ensayos de resistencia a compresión de las probetas de mortero de juntas, en los que se obtiene una resistencia a compresión media, $f_{m}$ de: $f_{m}=12 \mathrm{~N} / \mathrm{mm}^{2}$.

Como conclusión de los ensayos de mortero, que nos van a servir de base para la ejecución de los muretes de ladrillo cerámico, podemos decir que existe una cierta dispersión de resultados debido al sistema de realización a pie de obra.

No se consigue, en la práctica, unas dosificaciones suficientemente ajustadas a los parámetros que se prescriben en la norma UNE-EN 1015-11. Métodos de ensayo para morteros de fábrica. Por tanto, es fundamental para este tipo de fábricas estructurales, la utilización de morteros específicos, ya preparados, y con dosificaciones adecuadas (morteros predosificados).

\section{Muretes de fábrica}

Se construyen de acuerdo al plano reproducido en la Figura 2.

Se realizan 10 muretes con dos amasadas distintas de acuerdo con la norma UNE-EN 1052-1. Métodos de ensayo para albañilería. Parte 1: Determinación de la resistencia a compresión, y se ensayan a 28 días en una presa de 500 toneladas de capacidad, a la que se adapta una placa de reparto, como se muestra en la Figura 3 (14).

El resultado de los ensayos de compresión se especifican en la Tabla 3.

Con los resultados de rotura a compresión axial de los 10 muretes se procede a la obtención de la resistencia a compresión característica de la fábrica, de acuerdo a la norma UNE-EN 1052-1 cuyo proceso se expone en Tabla 4 , y en donde aparecen las siguientes variables:

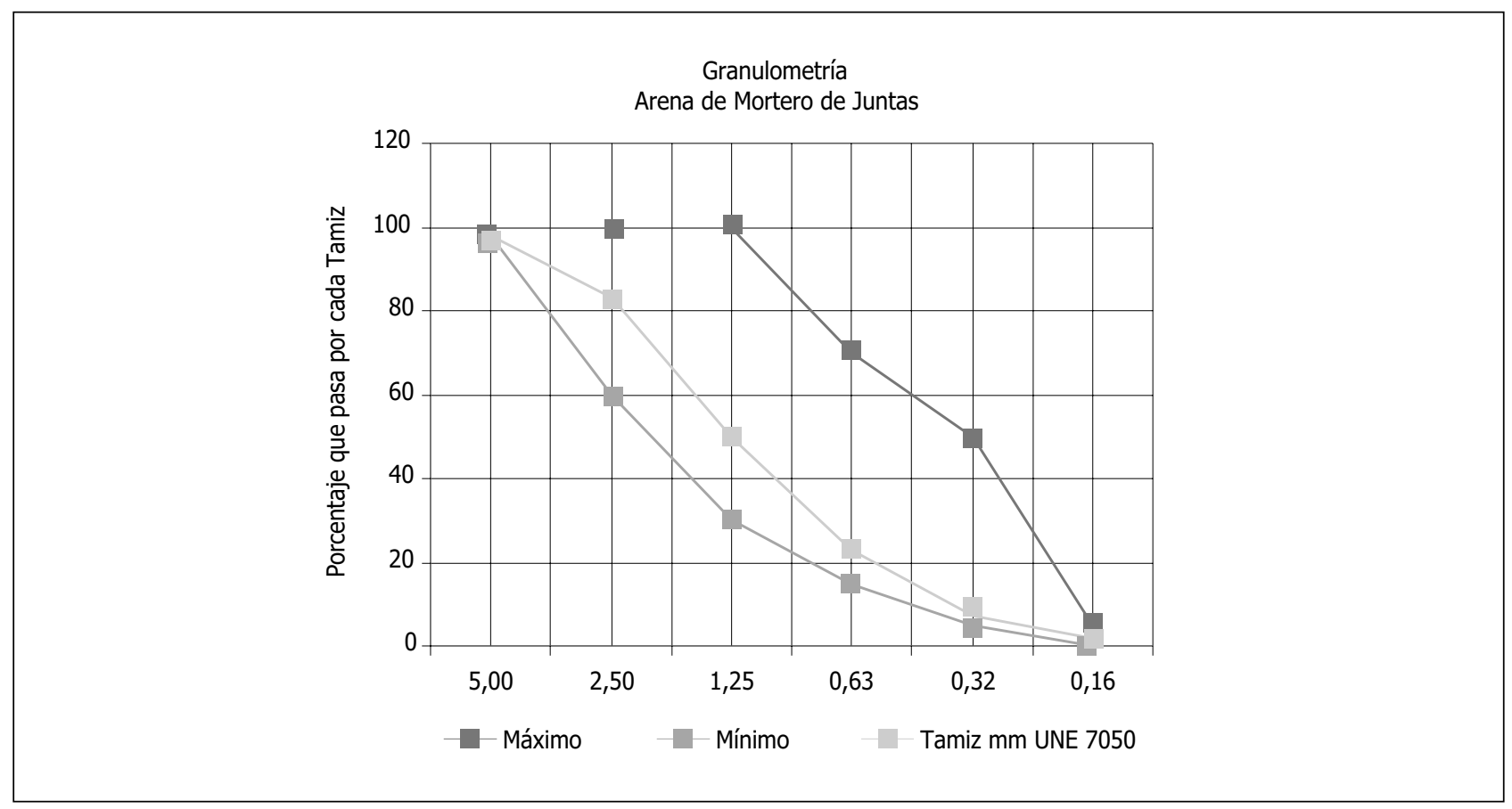

Figura 1. Análisis granulométrico de la arena empleada en la fabricación de los morteros de juntas. 
Tabla 2

Resultado de los ensayos a compresión, según la norma UNE-EN

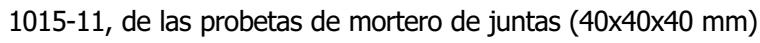

\begin{tabular}{|c|c|c|}
\hline Carga (kg) & & Tensión $\left(\mathrm{N} / \mathrm{mm}^{2}\right)$ \\
\hline 1.800 & & 11,48 \\
\hline 1.750 & & 11,16 \\
\hline 1.900 & & 12,11 \\
\hline 2.000 & & 12,75 \\
\hline 1.950 & & 12,43 \\
\hline \multirow[t]{2}{*}{2.100} & & 13,39 \\
\hline & V. Medio & 12,22 \\
\hline 1.041 & & 6,64 \\
\hline 1.313 & & 8,37 \\
\hline 1.052 & & 6,71 \\
\hline 1.073 & & 6,84 \\
\hline 1.005 & & 6,41 \\
\hline \multirow[t]{2}{*}{956} & & 6,10 \\
\hline & V. Medio & 6,84 \\
\hline 2.850 & & 18,17 \\
\hline 2.600 & & 16,58 \\
\hline 2.800 & & 17,86 \\
\hline 2.700 & & 17,22 \\
\hline 2.400 & & 15,30 \\
\hline \multirow[t]{2}{*}{2.500} & & 15,94 \\
\hline & V.Medio & 16,84 \\
\hline 1.700 & & 10,84 \\
\hline 1.500 & & 9,56 \\
\hline 1.800 & & 11,79 \\
\hline 1.750 & & 11,16 \\
\hline 1.500 & & 9,56 \\
\hline \multirow[t]{2}{*}{1.750} & & 11,16 \\
\hline & V. Medio & 10,68 \\
\hline 2.100 & & 13,39 \\
\hline 1.800 & & 11,47 \\
\hline 2.000 & & 12,75 \\
\hline 1.950 & & 12,43 \\
\hline 2.100 & & 13,39 \\
\hline \multirow[t]{3}{*}{1.950} & & 12,47 \\
\hline & V. Medio & 12,65 \\
\hline & V. Medio Total & 12,00 \\
\hline
\end{tabular}

$\mathrm{f}_{\mathrm{k}}$ - resistencia característica de la fábrica de ladrillo,

$f_{m}$ - resistencia media de la fábrica de ladrillo,

k - coeficiente en función del número de muretes ensayados. En este caso tiene el valor de 1,83 que se obtiene en la norma EN 1052-1,

s - es la desviación típica estimada.

Por tanto, la resistencia característica a compresión resultante es: $f_{k}=5,76 \mathrm{~N} / \mathrm{mm}^{2}$.
Tabla 3

Resultado de los ensayos de compresión de los muretes

\begin{tabular}{|c|c|}
\hline Muretes & Carga rotura - Ensayo - (t) \\
\hline 1 & 45,00 \\
\hline 2 & 55,00 \\
\hline 3 & 43,00 \\
\hline 4 & 40,00 \\
\hline 5 & 45,50 \\
\hline 6 & 42,00 \\
\hline 7 & 35,50 \\
\hline 8 & 33,00 \\
\hline 9 & 41,00 \\
\hline 10 & 43,50 \\
\hline
\end{tabular}

\section{RESULTADOS}

A continuación se procede a comparar los valores experimentales obtenidos para la resistencia característica de la fábrica, con los analíticos que se obtienen aplicando las expresiones de la norma UNE-EN 1996-1-1 Eurocódigo 6. Parte 1-1, en sus dos versiones, 1993 que aparece en la Figura 4, y 2003 que aparece en la Figura 5.

Con los valores:

$\mathrm{K}=0,55$, que corresponde al tipo de ladrillo, aparejo y espesor de la junta utilizado,

$f_{b}=16 \mathrm{~N} / \mathrm{mm}^{2}$, que corresponde con la resistencia normalizada a compresión del ladrillo empleado (obtenido en la Tabla 1),

$f_{m}=12 \mathrm{~N} / \mathrm{mm}^{2}$, que corresponde con la resistencia a compresión media del mortero empleado (obtenido en la Tabla 2),

y utilizando la gráfica de la Figura 4 entramos en las abscisas con el valor $f_{b}=16 \mathrm{~N} / \mathrm{mm}^{2}$, ascendemos en la vertical hasta encontrarnos con la curva correspondiente al $f_{m}=12 \mathrm{~N} / \mathrm{mm}^{2} \mathrm{y}$, recorriendo horizontalmente hacia la izquierda, obtenemos en las ordenadas el valor:

$f_{k}=\mathrm{Kf}_{\mathrm{b}} 0,65 \mathrm{f}_{\mathrm{m}} 0,25=6 \mathrm{~N} / \mathrm{mm}^{2}$, que es ligeramente superior al valor experimental $f_{k}=5,76 \mathrm{~N} / \mathrm{mm}^{2}$

Utilizando la gráfica de la Figura 5, entramos en las abscisas con el valor $f_{b}=16 \mathrm{~N} / \mathrm{mm}^{2}$, ascendemos en la vertical hasta encontrarnos con la curva correspondiente al $f_{m}=12 \mathrm{~N} / \mathrm{mm}^{2} \mathrm{y}$, recorriendo horizontalmente hacia la izquierda, obtenemos en las ordenadas el valor:

$f_{k}=\mathrm{K} \mathrm{f}_{\mathrm{b}} 0,70 \mathrm{f}_{\mathrm{m}}{ }^{0,30}=8 \mathrm{~N} / \mathrm{mm}^{2}$, que es bastante superior al valor experimental $f_{k}=5,76 \mathrm{~N} / \mathrm{mm}^{2}$ 


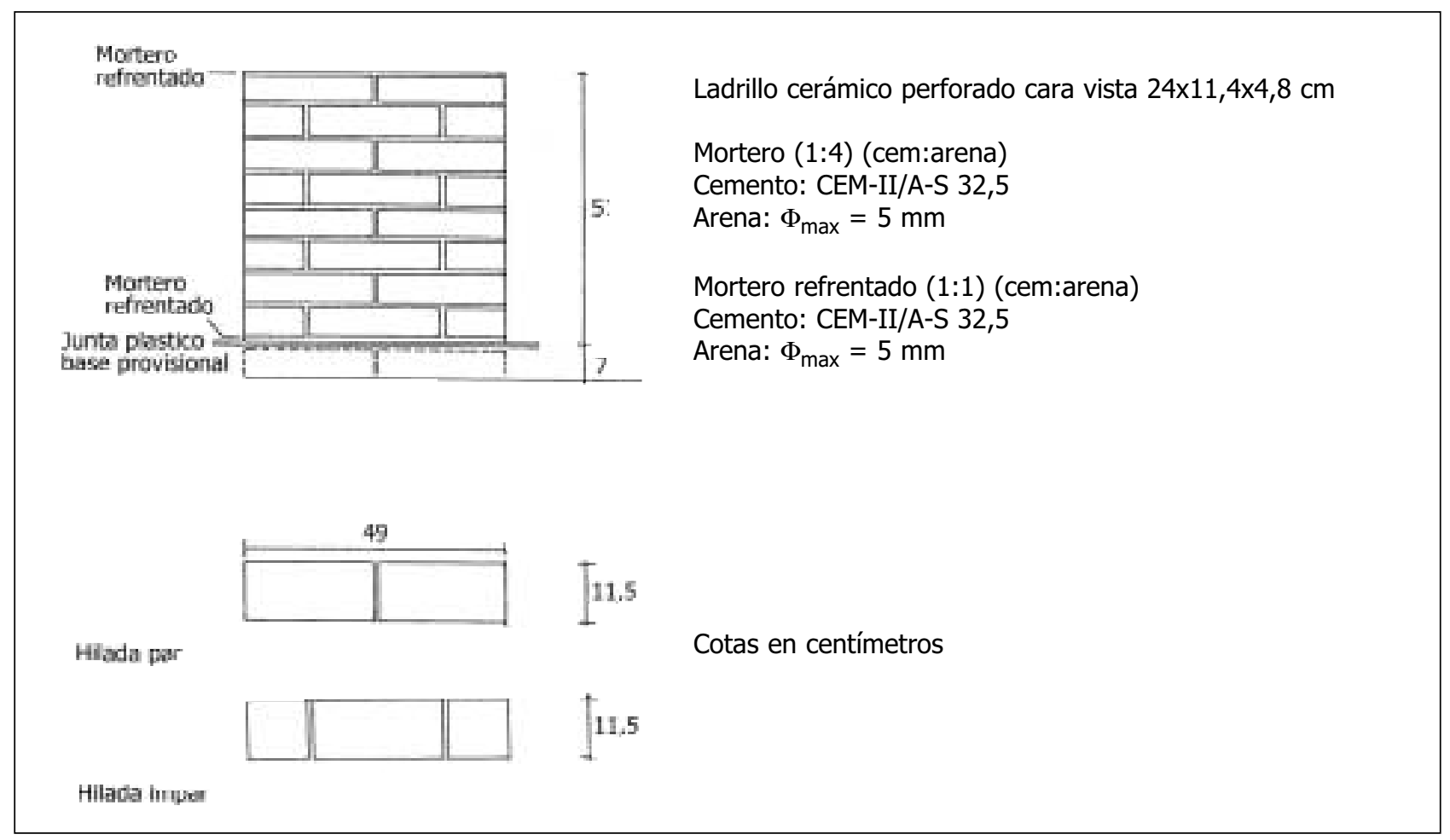

Figura 2. Plano de los muretes construidos.
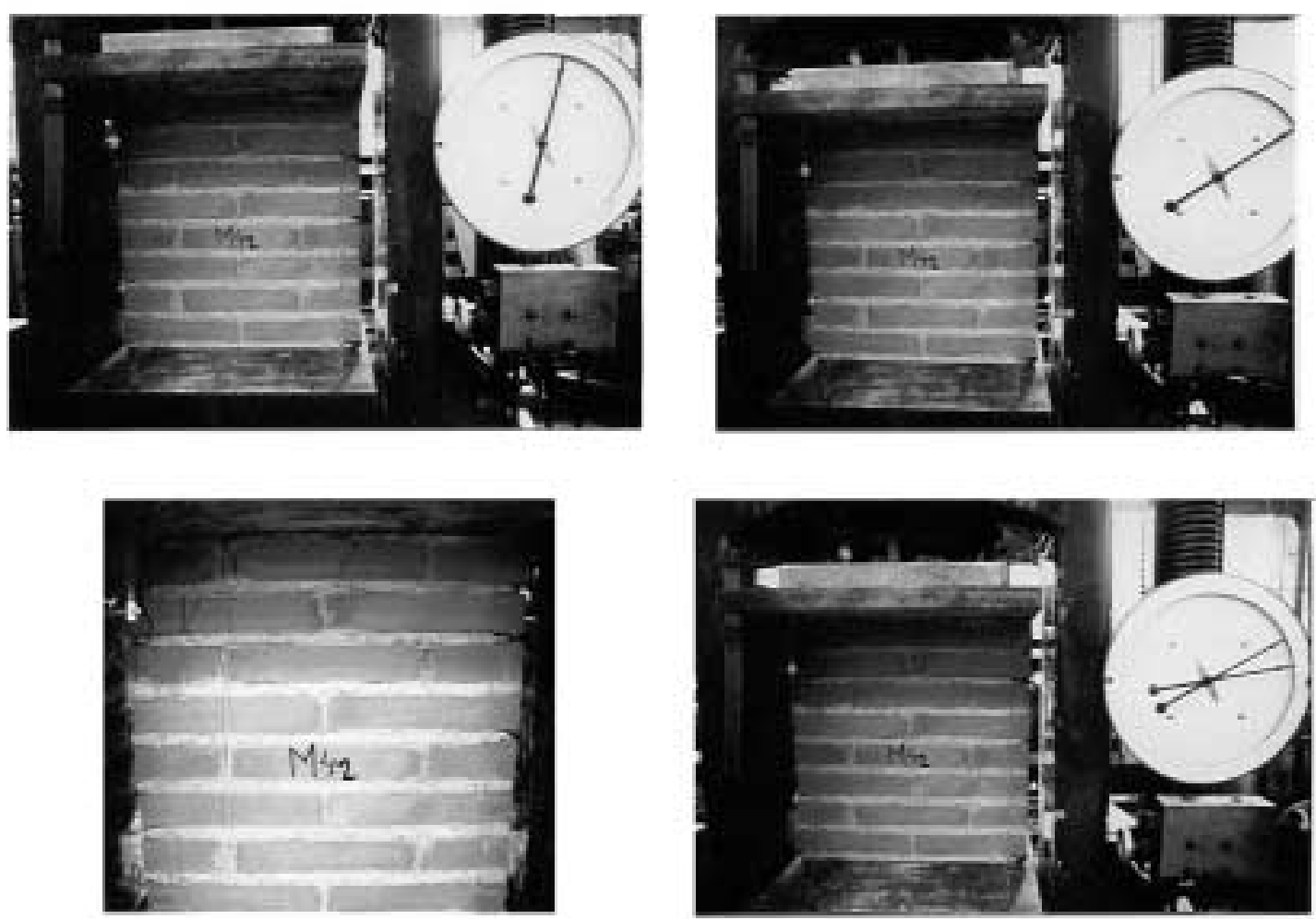

Figura 3. Vistas del ensayo de compresión axial de los muretes de fábrica. 
Tabla 4

Cálculo de la resistencia a comprensión característica de la fábrica de ladrillo

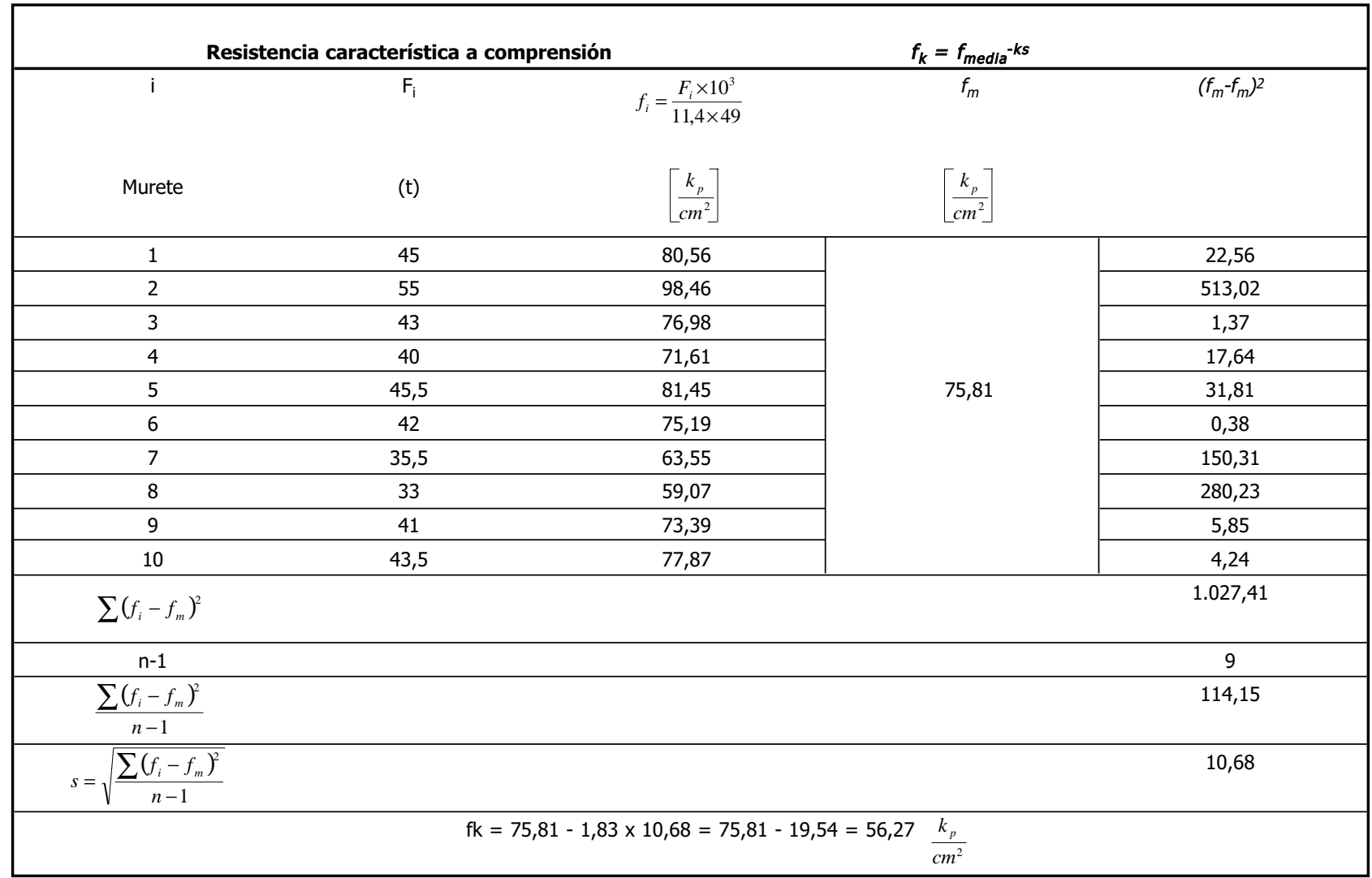

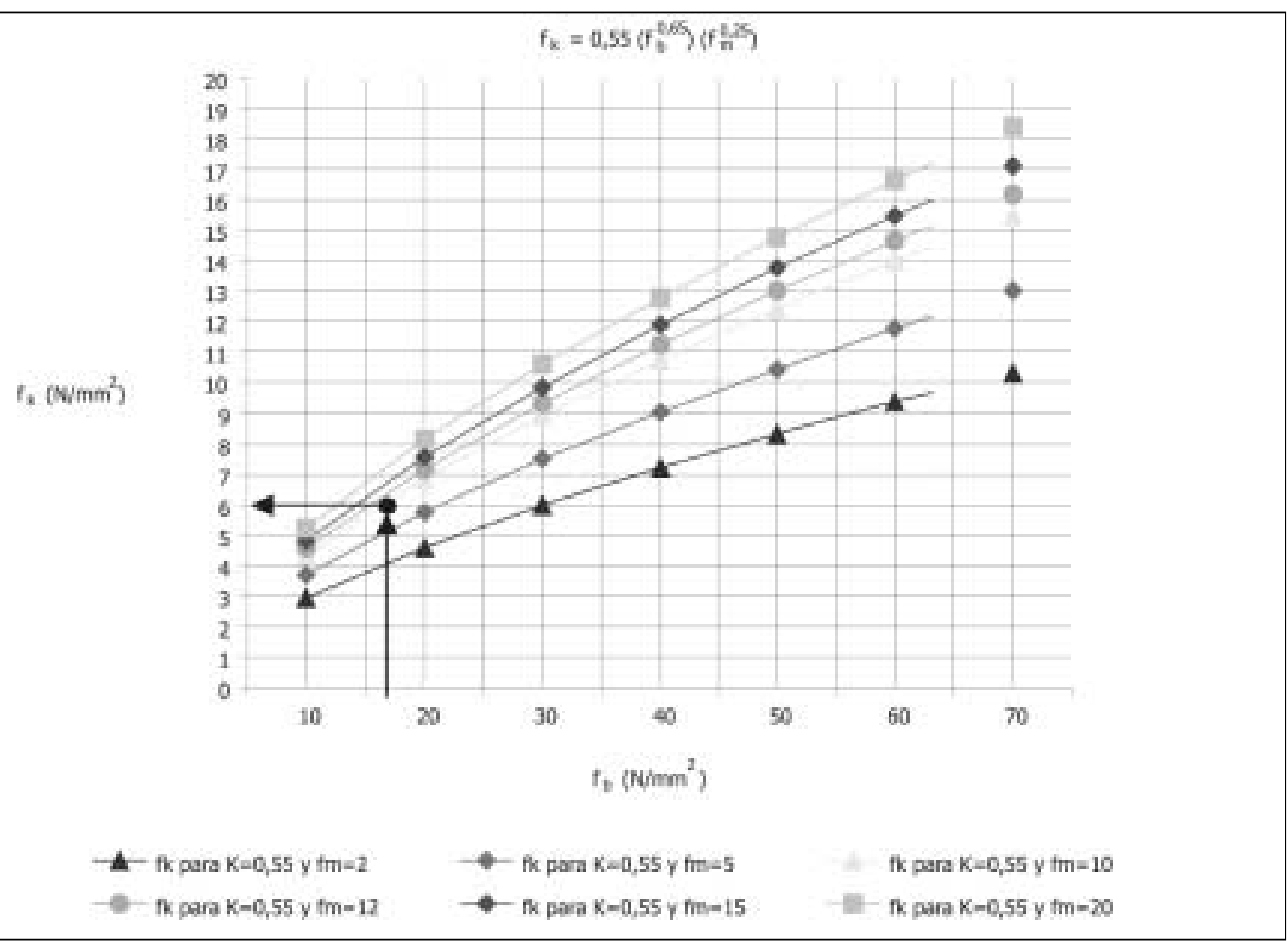

Figura 4. Representación de $f_{k}$ frente a $f_{b}$ de acuerdo a la norma UNE-EN 1996-1-1 Eurocódigo 6. Parte 1-1. Versión 1993. 


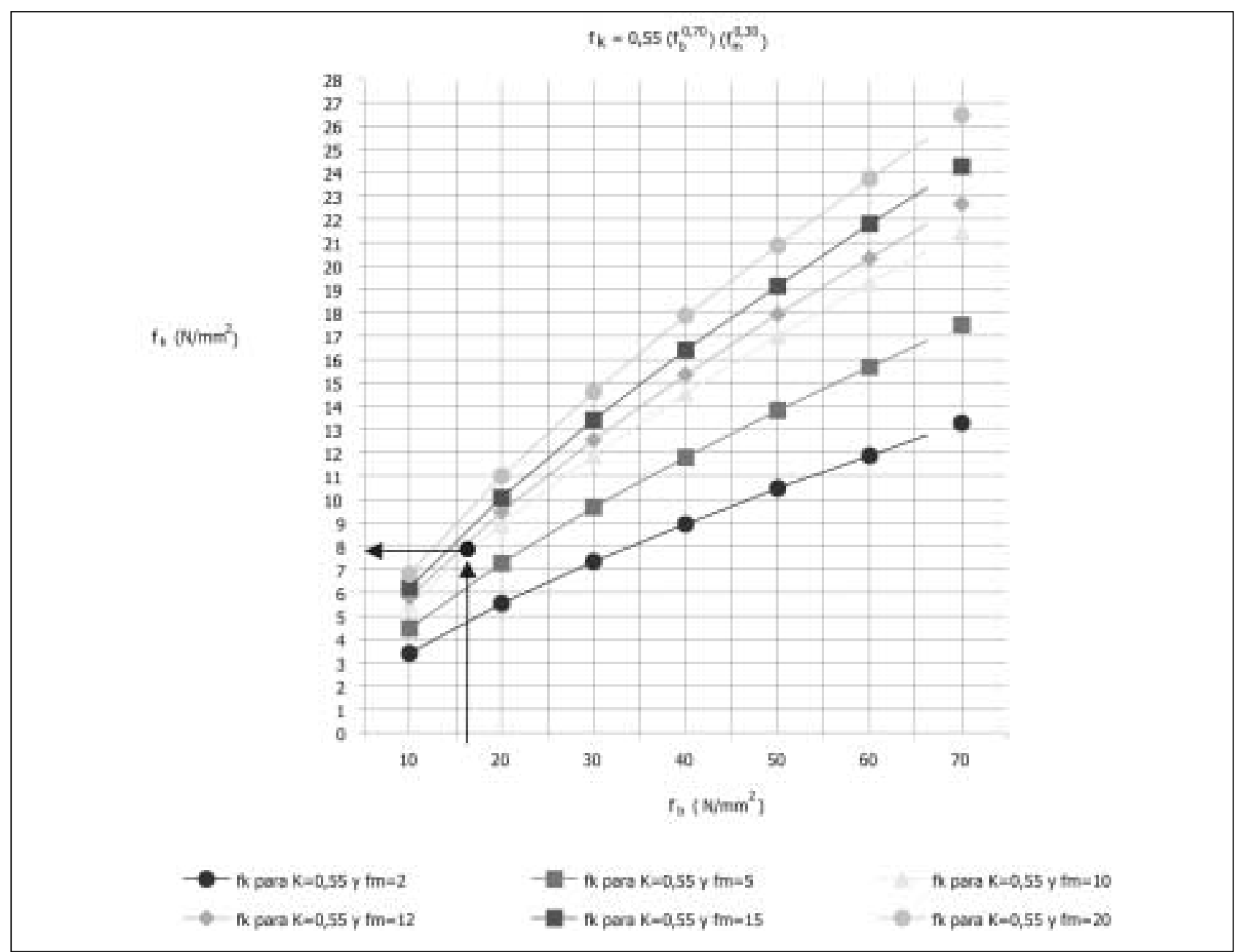

Figura 5. Representación de $f_{k}$ frente a f $f_{b}$ de acuerdo a la Norma UNE-EN 1996-1-1 Eurocódigo 6. Parte 1-1. Versión 2003.

\section{CONCLUSIONES}

\subsection{De los morteros}

Como conclusión de los ensayos de mortero que nos han servido de base para la ejecución de los muretes de ladrillo cerámico, podemos decir que existe una cierta dispersión de resultados debido al sistema de realización a pie de obra. No se consigue en la práctica unas dosificaciones suficientemente ajustadas a parámetros que se prescriben en la norma UNE-EN 1015-11. Métodos de ensayo para morteros de fábrica. Por tanto, es fundamental para este tipo de fábricas estructurales, la utilización de morteros específicos, ya preparados, y con dosificaciones adecuadas (morteros predosificados).

Se considera necesario el empleo de amasadora planetaria Ibertest, modelo CIB-701, según norma UNE 80101-88, para conseguir una buena mezcla de los componentes del mortero como mejora de la ejecución manual. Para la amasada de morteros, el conglomerante en polvo se mezclará en seco con la arena, añadiendo después el agua. El amasado se realizará preferentemente con amasadora planetaria Ibertest, modelo CIB-701, según norma UNE 80-101-88, batiendo el tiempo preciso para conseguir su uniformidad con un mínimo de 1 minuto. El amasado a mano no es recomendable (3).

\subsection{De los muretes de fábrica}

La expresión de $\mathrm{f}_{\mathrm{k}}$ representada en la gráfica de la Figura $4, f_{k}=K f_{b} 0,65 f_{m}{ }^{0,25}$ se ajusta más a los valores de los componentes ensayados habituales en la construcción de fábrica de ladrillo cerámico en España, que a la expresión $\mathrm{f}_{\mathrm{k}}$ dada en la última versión del Eurocódigo- 6 que aparece representada en la gráfica de la Figura $5, \mathrm{f}_{\mathrm{k}}=\mathrm{Kf}_{\mathrm{b}} 0,70 \mathrm{f}_{\mathrm{m}} 0,30$.

Habría que introducir en el Eurocódigo las condiciones de ejecución para tener en cuenta la dispersión de los valores en el mortero. 


\section{BIBLIOGRAFÍA / BIBLIOGRAPHY}

(1) Arredondo, F.: "La resistencia de los muros de ladrillo", Instituto Técnico de la Construcción y del Cemento (ITCC). Monografía no 173 (1957), pp. 3-15.

(2) Lahuerta, J.; Rodríguez, L. F.: "Normas técnicas de los ladrillos". Ministerio de la Vivienda. Secretaría Técnica, 1961 / "Muros de fábrica de ladrillo". Ministerio de la Vivienda. Secretaría Técnica, 1962.

(3) Cassinello, F.: "Arcos de ladrillo", Manuales y normas del ITCC, Madrid, 1958 / "Los amigos del ladrillo", ITCC-Monografía no 205, 1960 / "Muros de carga de fábrica de ladrillo", Instituto Eduardo Torroja, Monografía n 238, 1964 / "El ladrillo y sus fábricas", Instituto Eduardo Torroja, Manuales 4a edición, 1971.

(4) Instituto Eduardo Torroja: "PIET-70: obras de fábrica". Prescripciones del Instituto Eduardo Torroja (1971), Madrid, pp. 58-61.

(5) Norma NBE FL-90. Muros resistentes de fábrica de ladrillo (1991), pp. 206-207 (BOE no 4 de 4 de enero).

(6) Rolando Ayuso, A.: La fábrica de ladrillo armada: una nueva tecnología aplicada a un material tradicional, Editorial Rueda, Madrid, 1992, pp. 21-24.

(7) Th. Vermeltfoort, A.: "Compression properties of masonry and its components", Proceedings of the 10th international brick and block masonry conference. Calgary, vol. 3 (1994), pp. 1433-1442.

(8) Norma UNE-ENV 1996-1-1: "Eurocódigo 6. Proyecto de estructuras de fábrica. Parte 1-1: reglas generales para edificios. Reglas para fábrica y fábrica armada", 2003.

(9) Norma UNE-EN 771-1: "Especificación para las piezas de fábrica. Parte 1: Piezas cerámicas".

(10) Norma UNE-EN 772-1: "Métodos de ensayo para piezas de fábrica. Parte 1: Determinación de la resistencia a compresión".

(11) Norma UNE-EN 998-2: "Especificaciones de los morteros para albañilería. Parte 2: Morteros para albañilería".

(12) Norma UNE-EN 1015-11: Métodos de ensayo de los morteros para albañilería. Parte 11: Determinación de la resistencia a flexión y a compresión del mortero endurecido".

(13) Norma UNE-EN 1015-2: "Métodos de ensayo de los morteros para albañilería. Parte 2: Toma de muestras total de morteros y preparación de los morteros para ensayo".

(14) Norma UNE-EN 1052-1: "Métodos de ensayo para albañilería. Parte 1: Determinación de la resistencia a compresión".

(15) Norma BS 5628: "Parte 1. Utilización estructural de la fábrica no armada". 